\title{
The use of the two-body energy to study problems of escape/capture
}

\author{
Ernesto Vieira Neto ${ }^{1}$, O.C. Winter ${ }^{1,2}$ and C.F. Melo ${ }^{2}$ \\ ${ }^{1}$ Grupo de Dinâmica Orbital e Planetologia - UNESP $\dagger$. \\ email: ernesto@feg.unesp.br \\ ${ }^{2}$ Instituto Nacional de Pesquisas Espaciais.
}

\begin{abstract}
The problem of escape/capture is encountered in many problems of the celestial mechanics - the capture of the giants planets irregular satellites, comets capture by Jupiter, and also orbital transfer between two celestial bodies as Earth and Moon. To study these problems we introduce an approach which is based on the numerical integration of a grid of initial conditions. The two-body energy of the particle relative to a celestial body defines the escape/capture. The trajectories are integrated into the past from initial conditions with negative two-body energy. The energy change from negative to positive is considered as an escape. By reversing the time, this escape turns into a capture. Using this technique we can understand many characteristics of the problem, as the maximum capture time, stable regions where the particles cannot escape from, and others. The advantage of this kind of approach is that it can be used out of plane (that is, for any inclination), and with perturbations in the dynamics of the n-body problem.
\end{abstract}

Keywords. Celestial mechanics, planets and satellites: general.

\section{Introduction}

A precise definition of gravitational capture is not yet established. There are several papers which define some kind of capture, as Heppenheimer (1975) with the passage of the particle by the inner Lagrangian point $\left(L_{1}\right)$. In this work the definition given by Yamakawa (1992) is adopted, and it reads: if the local two-body energy of the particle relative to a celestial body changes from positive (hyperbolic motion) to negative (elliptic motion) it is considered a capture. The local two-body energy can be formulated as:

$$
C 3=v^{2}-\frac{2 \mu}{r}
$$

where $v$ is the velocity of the particle relative to the celestial body, $\mu$ is the mass parameter of the body, and $r$ is the distance from the body to the particle.

In the two-body problem $C 3$ is constant, but any perturbation to the two-body problem will change this energy. For negatives values of $C 3$ the orbit of a particle is closed (elliptic motion), for positives values the orbit is open (hyperbolic motion). Thus, in the course of the integration of the particle's orbit, we monitor the value of $C 3$ and when a change of sign occurs, we record an escape or a capture.

In the restricted three-body problem, if a particle has a Jacobi constant $C_{J}$ greater than the $C_{J}\left(L_{1}\right)$, and it is in the vicinity of the secondary, $C 3$ will vary, but never assume a positive value. Thus this particle will never escape from the secondary, as expected. If the particle has $C_{J}$ smaller than $C_{J}\left(L_{1}\right)$ (the bottle neck near $L_{1}$ is open), and it is in the vicinity of the secondary, it can have a negative value of $C 3$, which may vary to a positive value at some time, that is, it can escape (see Figure 1).

$\dagger$ Address: Av. Ariberto Pereira da Cunha, 333, CEP 12516-410, Guaratinguetá, S.P. Brazil. 


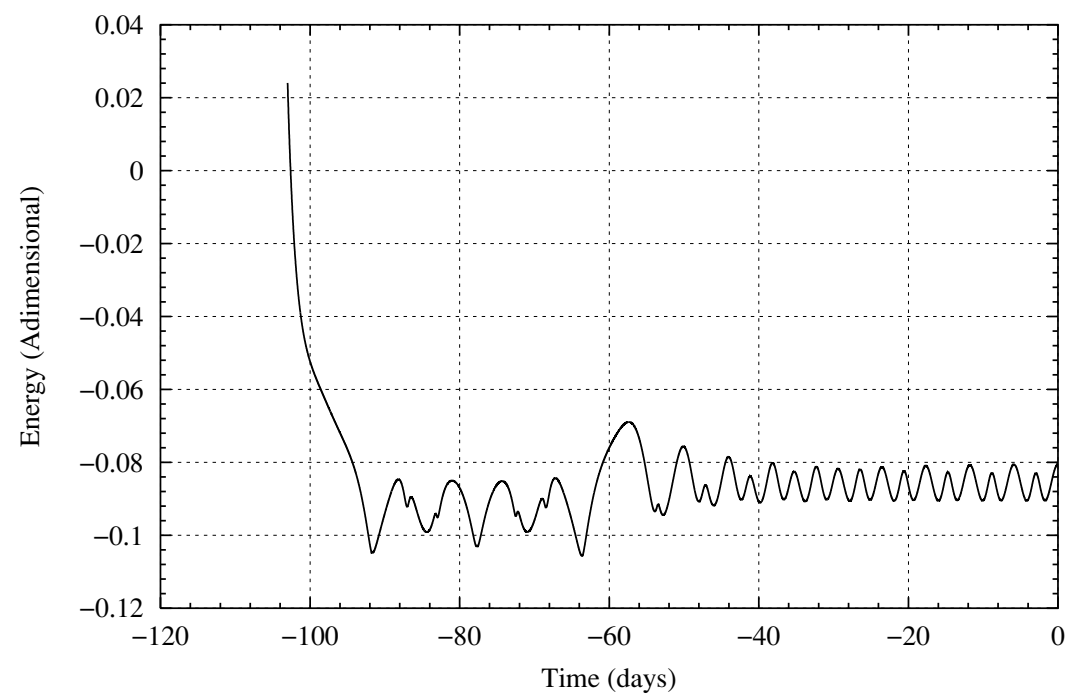

Figure 1. Variation of the energy $(C 3)$ for the restricted three body problem. The energy of the particle is measured relative to the secondary body (or planet).

We use this simple rule to study regions near a celestial body. There are other tools to make this kind of study, such as Poincaré Surface of section (see for example Hénon 1970), but they have some limitations. For example, it is very difficult to use the Poincaré Surface of Section to study the four-body problem, or inclined orbits in the three-body problem.

\section{The method}

To use this method it is necessary to choose a region in the vicinity of the celestial body, the variables to represent the region, and the dynamical model to be used. For example, the orbital elements of a particle relative to a planet can be used to study the stability of the trajectories. Orbits with eccentricities lower than one (elliptic orbits) have negative two-body energy; if we introduce another planet (or the Sun) the perturbations on the trajectory can make the particle crash into the planet, or escape from the planet. We define these trajectories as unstable, to distinguish them from the others which stay around the planet.

As an example, lets study a region around the Moon. The dynamical model was the circular restricted three-body problem. The variables were the selenocentric orbital elements. The initial values of the angular variables were fixed at zero, that is $i=0^{\circ}, \Omega=0^{\circ}, \omega=0^{\circ}, M=0^{\circ}$. The value of the semi-major axis and the eccentricity varied from $20000 \mathrm{~km}$ to $35000 \mathrm{~km}$, and from 0.00 to 0.99 , respectively. The trajectories were integrated from time $t=0$ to $t=-5000$ days. The integration was made to the past because in reversing the time the escape becomes a capture. The result for this grid is shown in Figure 2 where the gray scale is associated with the escape time, that is, the time when $C 3$ changes its sign from negative to positive.

On the left hand side of Figure 2 there is a region in white whose trajectories have the $C_{J}$ value greater than $C_{J}\left(L_{1}\right)$. As expected, these trajectories do not escape in the time span covered by the integration. However, there are some trajectories which do not escape, but have $C_{J}$ value smaller than $C_{J}\left(L_{1}\right)$; these are the trajectories on the right hand side of the curve $C_{J}=C_{J}\left(L_{1}\right)$. These trajectories are associated with the family of 


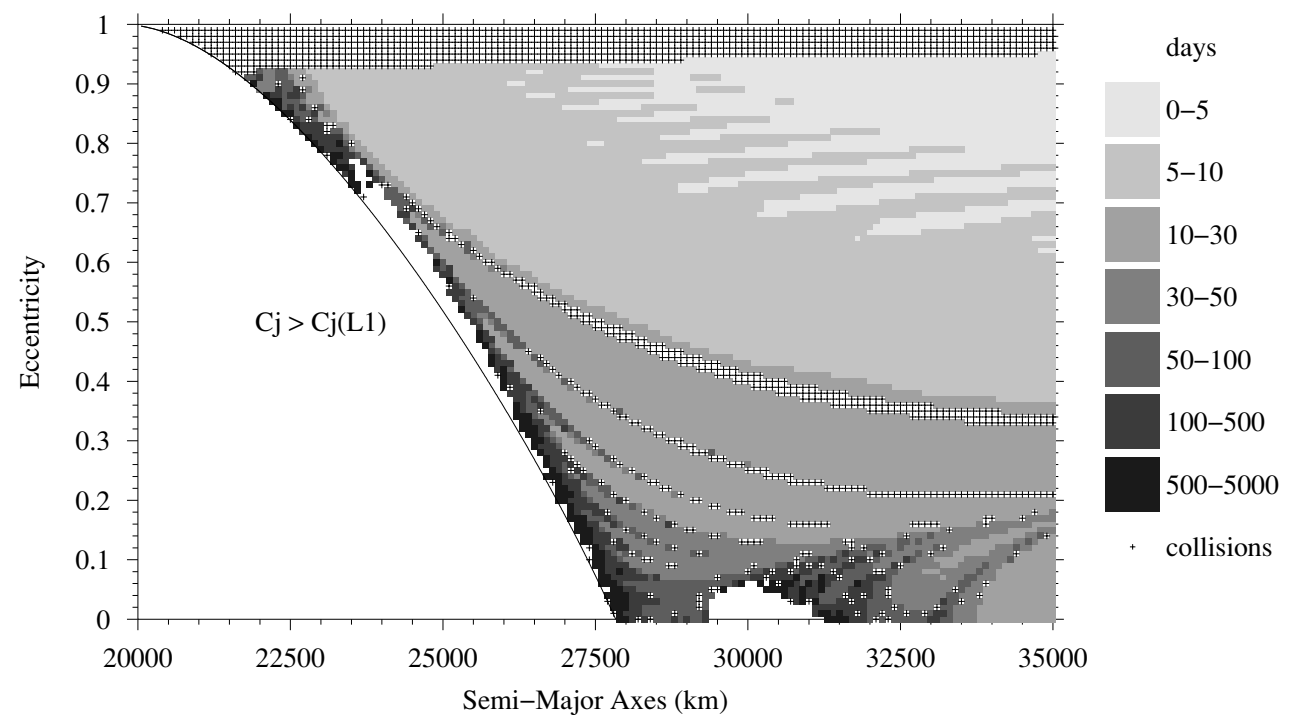

Figure 2. Study of the stable region around the Moon. The escape time is shown in gray scale. Each point is a trajectory with initial conditions $i=0^{\circ}, \Omega=0^{\circ}, \omega=0^{\circ}, M=0^{\circ}$, and $a$ and $e$ as on the axes. The white regions are trajectories that did not escape in 5000 days.

direct periodic orbits denoted $h_{1}$ by Broucke (1968) (or family $g_{1}$ by Hénon 1970). The other white region, with semi-major axis about $30000 \mathrm{~km}$ is associated with family $h_{2}$ by Broucke (1968) (or $g_{2}$ of Hénon 1970).

There are other remarkable characteristics in Figure 2. For example, on the top we see a region with crosses, this region is filled with trajectories which collide with the Moon due to the high initial eccentricity of the orbits. It is also possible to see these collision lines in the figure with trajectories with low initial eccentricity.

The $h_{2}$ family is better seen when we change the pericenter to $\omega=180^{\circ}$, as it is shown in Figure 3. In Figure 2 the particles have initial conditions at opposition with respect to the Moon and in Figure 3 the trajectories initiate at conjunction with respect to the Moon. Thus, with simple change of the initial values of the relevant variable we can have better information about the characteristics of the region.

In Winter \& Vieira Neto (2002) these regions were discussed in detail. In that paper the Poincaré Surface of Section method was used, and it was shown that the white regions are associated with periodic orbits.

As stated before, the advantage of the two-body energy method is the possibility to study regions out of plane and to use more complex dynamics. In Figure 4 we explore this possibility by using the four-body dynamical model to study the stability of the same region as of Figure 3. In this case Earth, Moon and Sun interact with each other, but all moving in the same plane. Here it is not possible to make use of the usual Poincaré Surface of Section method because there is not a Jacobi constant for this problem, thus only the use of the two-body energy in this problem can reveal the location of the trajectories which will not escape in the time span of integration. Although the area of stability is reduced in Figure 4, we see that the solar perturbation on the region does not destroy it.

With the two-body energy method it is also possible to study other effects on the trajectories, like the effect of the Moon's orbital inclination, or that of the Earth's and the Moon's orbital eccentricities, one can use dynamical models with variation of mass in the three-body problem, as in Vieira Neto et al. (2004). In this case, due to the change of the gravitational influence of the planet, the particles integrated with a negative step 


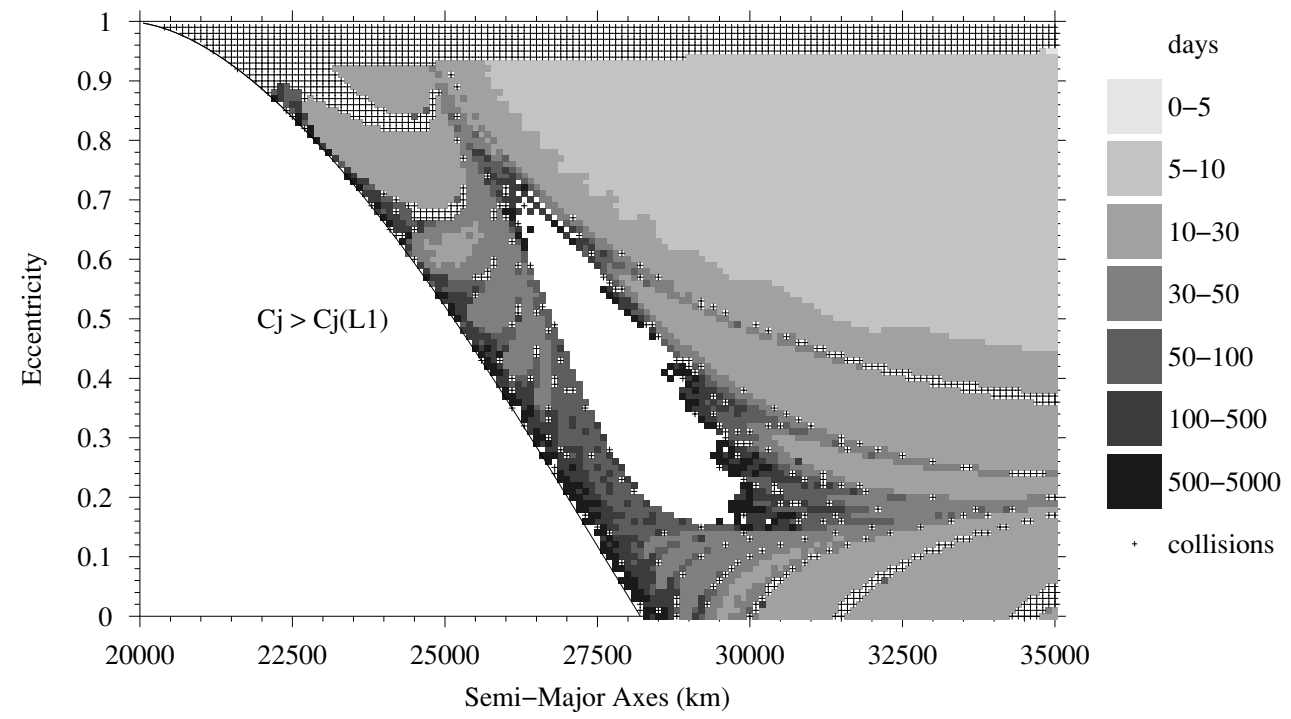

Figure 3. Study of the stable region around the Moon. The escape time is shown in gray scale. Each point is a trajectory with initial conditions $i=0^{\circ}, \Omega=0^{\circ}, \omega=180^{\circ}, M=0^{\circ}$, and $a$ and $e$ as on the axes. The white regions are trajectories that did not escape in 5000 days.

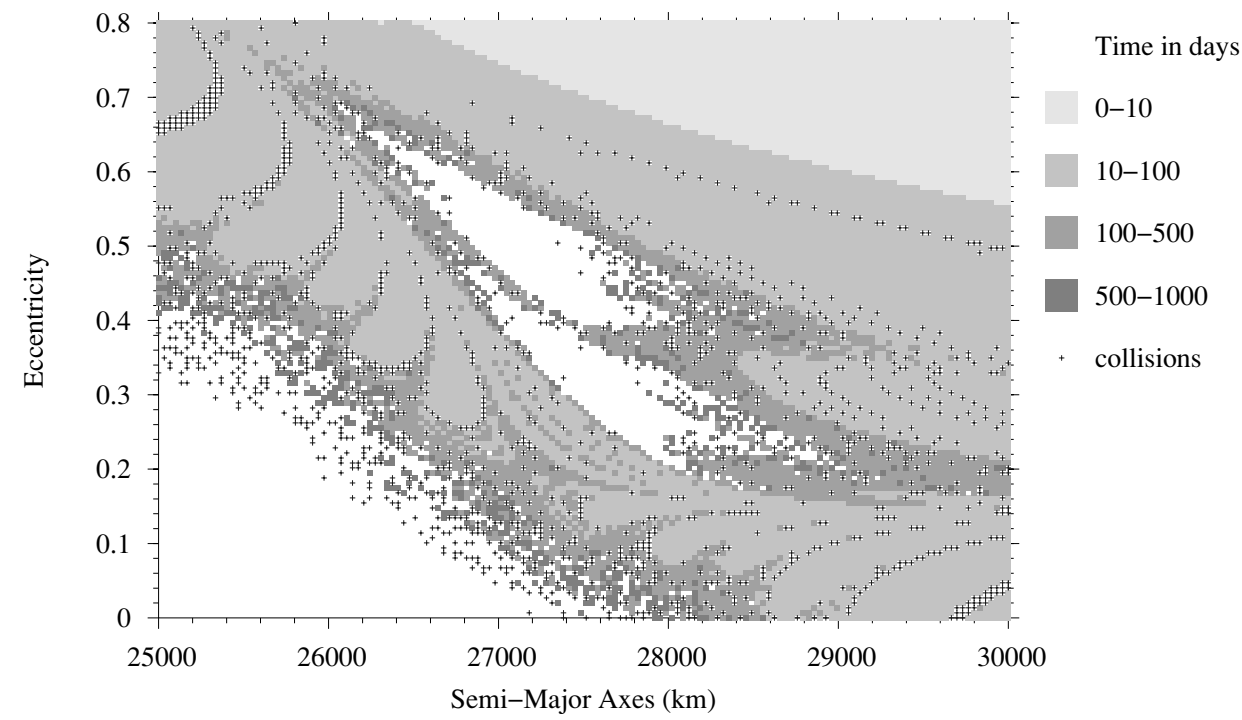

Figure 4. Study of the stable region around the Moon. Each point is a trajectory with initial conditions $i=0^{\circ}, \Omega=0^{\circ}, \omega=180^{\circ}, M=0^{\circ}$, and $a$ and $e$ as on the axes. The white regions are trajectories that did not escape in 5000 days. The dynamical model used in this case is the four body problem with the particle, Earth, Moon and the Sun.

in time escaped. In this kind of analysis, the mass of the planet when the escape occurs is used as indicative for the analysis instead of the escape time. Reversing the time we found that particle in heliocentric orbit approached the planet and, due to the mass variation of the planet, became a satellite.

Another approach is to study the lowest energy of the capture around the Moon. In Figure 5 we vary the energy and the pericenter distance of the particle relative to the Moon and we measure the time for a trajectory to escape (Winter et al. 2003). As in 


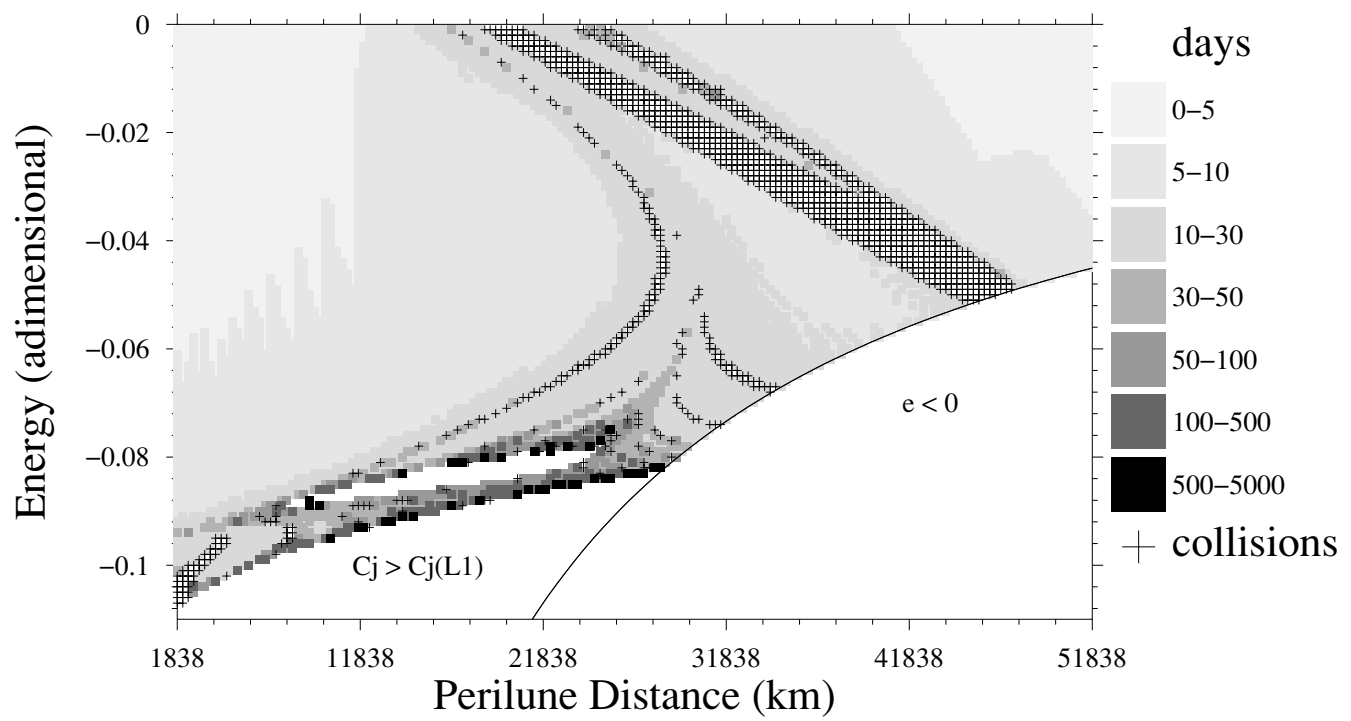

Figure 5. The minimum orbital energy of a particle as a function of the perilune position. The gray scale represents the time, in days, that the trajectory takes to escape from the Moon. The pericenter is pointed towards the Earth.

the previous figures, there is a region where the particle can not escape from the Moon due to the value of the Jacobi constant. In the lower right corner of the figure there is a region where the eccentricity of the particle is negative for the given values of energy and pericenter distance. The time for the capture has an exponential growth, and the longest capture times are detected at the border defined by the value of the Jacobi constant $C_{J}\left(L_{1}\right)$. The white island inside the grey region, is a region where the trajectories do not escape during the integration time of 5000 days. Basically we have the same structures as on the other figures but this one gives us information of the lowest energy possible to achieve certain perilune distance and the time that the particle takes to achieve that position and energy.

\section{Conclusion}

The two-body energy of a particle, relative to some celestial body, can be used to study escape/capture problems. This approach can give us ample information as discussed in this work. This method have some difficulties, as the extensive consumption of computational time when integrating trajectories in the stable regions. This computational time depends on the total time used for the trajectory integration, and for some systems the integration time must therefore be long. It is, however, easier than with other methods to study the stability, and it can also be used in more general conditions such as out of the orbital plane and with dynamics which includes mass variation, or gas drag.

\section{Acknowledgements}

We would like to acknowledge the support of FAPESP under grant number 02/00344-7 and $\mathrm{CNPq}$. Also we are grateful to an anonymous referee for his useful comments. 


\section{References}

Broucke, R. A. 1968, JPL-NASA, Technical Report 32-1168

Hénon, M. 1970, Astron. Astrophys. 9, 24

Heppenheimer, T. A. 1975. Icarus 24, 172

Vieira Neto, E., Winter, O. C. and Yokoyama, T. 2004, Astron. Astrophys. 414, 727

Winter, O. C. and Vieira Neto, E. 2002, Astron. Astrophys. 393, 661

Winter, O.C., Vieira Neto, E. and Prado, A.F.B.A. 2003, Advances Space Res. 31, 2005

Yamakawa, H. 1992, PhD Thesis, University of Tokyo. 